\title{
Type-specific Prevalence of Human Papillomavirus by Cervical Cytology among Women in Brasov, Romania
}

\author{
Marius Alexandru Moga ${ }^{1}$, Marius Irimie ${ }^{2 *}$, Alexandru Oanta ${ }^{2}$, Alina Pascu ${ }^{3}$, \\ Victoria Burtea ${ }^{4}$
}

\begin{abstract}
The oncogenic role of human papillomavirus (HPV) in triggering cervical cancer, the second most common cancer in women worldwide, is well established. Romania ranks in first place in Europe in terms of the incidence of cervical cancer. Geographical widespread data on HPV type-distribution are essential for estimating the impact of HPV vaccines and cervical cancer screening programmes. In this study we aimed to identify the prevalence of HPV genotypes and to establish correlations with abnormal cervical cytology among the female population of Brasov County, Romania. A total of 1,000 women aged 17.3-57 years, attending routine cervical examination in the Obstetrics and Gynecology Hospital of Brasov, Romania, and undergoing both cytological examination and HPV genotyping were screened. Infection with 35 different HPV genotypes was detected in $39.6 \%$ of cytological specimens. Overall HPV infections were highest in young women under 25 years $(\mathbf{p}<0.0001)$, in which cervical cytological abnormalities also reached the highest prevalence. Patients infected by HPV-16 or HPV-18 showed the highest prevalence of cervical cytological abnormalities. Some $48.2 \%$ of women with abnormal cytology were infected with high-risk HPV types whereas less than $3 \%$ of them were infected only with low-risk HPV types. Our study showed that the prevalence of high-risk HPV infection among Romanian women is higher compared to other studies in other geographic areas. Thus, we consider that in areas where there is an increased prevalence of high-risk HPV infections, HPV genotyping should be performed in all women aged between 18 and 45 years, and Pap test should be performed every 6 months in women with high-risk HPV infection, even those with previous normal cervical cytology.
\end{abstract}

Keywords: Human papillomavirus - cervical cancer - genotyping - Brasov, Romania

Asian Pac J Cancer Prev, 15 (16), 6887-6892

\section{Introduction}

Genital infection with human papillomavirus (HPV) is the most common sexually transmitted infection in women (Aral; Holmes, 1999). According to the most recent classification there are $15 \mathrm{HPV}$ genotypes defined as high-risk (HR-HPV) oncogenic (HPV 16, 18, 31, 33, $35,39,45,51,52,56,58,59,68,73$, and 82), 3 probable high-risk genotypes (PHR-HPV) (HPV 26, 53, 66), 10 low risk genotypes (LR-HPV) (HPV 6, 11, 40, 42, 54, $61,70,72,81$, and CP6108), and 9 genotypes for which the risk is still undetermined (UR-HPV)(HPV 55, 62, 64, 67, 69, 71, 83, 84, and IS39) (IARC, 2011; Halfon et al., 2013). About 50 of HPV types have a high tropism for the ano-genital mucosa and are sexually transmitted. Persistent infection with HR-HPV has been identified as the most important cause of cervical cancer (CC) (Clifford et al., 2003; Wang et al., 2013; Sengul et al., 2014). There are at least 15 oncogenic HR-HPV types that have been recognized as the cause of nearly all CC (Smith et al.,
2007; Bouvard et al., 2009).

As a result of numerous studies that have demonstrated the causal relationship between HPV and CC, guidelines for assessing risk patients, especially in women over 30 years, were developed (Davey et al., 2004; Wang et al., 2014). Most of them recommended the Pap smear as a screening test for evidence of cytological abnormalities (Nessa et al., 2013). However, the Pap smear has limited sensitivity in detecting cancer precursors, giving a false - negative rate ranging from 20 to $30 \%$ (Veena et al., 2012; Sengul et al., 2014). As an alternative to cervical cytology, HPV genotyping to identify HR-HPV in women with borderline pathology is recommended (Mandelblatt et al., 2002).

According to the International Agency for Research in Cancer GLOBOCAN 2008 CC is the second malignancy in women as frequency and the first in the age group 15-44 years. Romania ranks first in Europe in terms of incidence of CC. Annually 3,402 (23.9\%) new cases are diagnosed and 2,005 (10.6\%) deaths are recorded in this 
issue (Globocan, 2008).

The high frequency of HR-HPV types infections in CC, especially with HPV-16 and HPV-18, lead to the development of HPV vaccines against HPV-6, 11, 16, and 18 which have the potential to reduce the incidence of CC. HPV types distribution data is the basic need to predict the potential advantages of HPV immunization and develop more reliable CC screening tests. However, type-specific HPV prevalence has been shown to vary among populations according to age, region and the type of population (Clifford et al., 2005; De Sanjose et al., 2010). Varied distribution of HPVs across populations indicates the importance of HPV surveys in different geographical regions. The data on the prevalence of HPVs infection in Romania are not available.

The study aimed to assess the rate of genital HPV infection, to identify the prevalence of HPV genotypes and to establish correlations with abnormal cervical cytology among the female population of Brasov County attending routine gynecological visits.

\section{Materials and Methods}

\section{Study population}

Between January 2012 and May 2013, a total of 1000 consecutive women that attending the gynecology outpatient clinic of Brasov, Romania, for routine cervical examination and undergone both cytological examination and HPV genotyping were screened. The patients that exhibited acute inflammation of the reproductive tract were not included in study. The mean age of the participants was 32.73 \pm 7.69 years (range 17.3-57 years). The patients were divided by their age into four groups: $<25$ years $(n=193), 26-35$ years $(n=501), 36-45(n=257)$ and $>45$ years $(n=49)$.

\section{Clinical Specimens}

Epithelial cervical specimens from every patient were collected in PreservCyt (Cytec Corp, Boxborough, MA) solution and submitted to the laboratory for cytology test and HPV detection. All samples were analyzed in the pathology and molecular laboratory, respectively, of our clinic.

\section{Cytology test}

The cytology specimens were obtained by use of an endocervical cytobrush and an experienced pathologist evaluated the histopathological slides. The Bethesda system protocol, revised by the International Cancer Association in 2001, was used as the standard for cytological diagnosis, the results being classified into four categories: Negative for Intraepithelial Lesions or Malignancy (NILM), Atypical Squamous Cells of Undetermined Significance (ASCUS), Low grade Squamous Intraepithelial Lesions (LSIL), High grade Squamous Intraepithelial Lesions (HSIL).

\section{DNA extraction}

Automated HPV DNA extraction was done on the MagNA (MP) pure instrument by using the MagNA Pure LC Total Nucleic Acid Isolation kit (Roche Applied
Science, Mannheim, Germany), as described by the manufacturer. Prior to automated HPV DNA extraction on the MagNA Pure LC, $400 \mu$ l of the sample transport medium were centrifuged with 12,000 rpm for 5 minutes at room temperature. After discarding the supernatant, the precipitate was resuspended in $190 \mu$ l of tissue lysis buffer (Roche Applied Science) followed by addition of $10 \mu \mathrm{l}$ of Proteinase K (Roche Applied Science). After incubation on a mixing platform for 3 hours at $56^{\circ} \mathrm{C}$, the sample was transferred into the MagNA Pure LC reaction tube.

The DNA was isolated from $200 \mu$ l of a ViraPap/ Viratype aliquot by using the MP. A negative control was included in each DNA extraction run to monitor the DNA isolation procedure. DNA was resuspended in $120 \mu \mathrm{l}$ of PCR-grade $\mathrm{H}_{2} \mathrm{O}$, and stored at $-20^{\circ} \mathrm{C}$ until further processing by Linear Array Genotyping Test.

\section{LINEAR ARRAY}

All samples were examined using LINEAR ARRAY HPV Genotyping Test (CE-IVD), based on reverse hybridization of amplicons. The DNA of $37 \mathrm{HPV}$ types $(6$, $11,16,18,26,31,33,35,39,40,42,45,51,52,53,54,55$, $56,58,59,61,62,64,66,67,68,69,70,71,72,73,81,82$, 83,84 , IS39 and CP6108) was detected in cervical samples by multiplex PCR targeted to the conserved L1 region of the viral genome. The Gene Amp PCR System 9700 was used for genotyping test according to the manufacturer's instructions. Automated hybridization and detection of HPV DNA was done on the ProfiBlot 48 (Tecan Trading AG, Zurich, Switzerland).

\section{Reference Samples}

A panel of samples from an external quality assurance program, including HPV 16, 18, and 6, and a HPV negative control probe (MRC-5 cells) were used as reference (INSTAND, Düsseldorf, Germany).

\section{Statistical analysis}

Distribution of HPV infection according to age and cytological lesion types was analyzed. The data were analyzed by using SPSS 19.0 software. The baseline characteristics of continuous variables were compared using Student's t-test. Chi-square test was used for comparison of the prevalence between the different groups. Odds ratios (OR) and their $95 \%$ CI were calculated where appropriate. $\mathrm{p}<0.05$ were considered to indicate a statistically significant difference.

\section{Results}

Infection with 35 different HPV genotypes was detected in 396 of 1000 cytological specimens (39.6\%). Globally, 818 HPV including 417 HR-HPV specific-type infections were detected in the $396 \mathrm{HPV}$ positive women. All detected HPV types were stratified into the following four categories: 15 HR-HPV types (HPV 16, 18, 31, 33, 35, 39, 45, 51, 52, 56, 58, 59, 68, 73, and 82), 3 PHR-HPV types (HPV 26, 53, 66), 10 LR-HPV types (HPV 6, 11, 40, $42,54,61,70,72,81$, and CP6108), and 9 types for which the risk is still undetermined (UR-HPV) (HPV 55, 62, 64, $67,69,71,83,84$, and IS39). In total, the most prevalent 
Type-Specific Prevalence of Human Papillomavirus by Cytology in Romania

Table 1. Distribution of HPV Genotypes and Cytological Diagnosis by Age

\begin{tabular}{|c|c|c|c|c|c|}
\hline Age group & $<25$ years & 26-35 years & $36-45$ years & $>45$ years & Total $(\%)$ \\
\hline Number of samples & $193(19.3 \%)^{t}$ & $501(50.1 \%)^{\mathrm{t}}$ & $257(25.7 \%)^{\mathrm{t}}$ & $49 \quad(4.9 \%)^{\mathrm{t}}$ & $1000(100 \%)^{t}$ \\
\hline HPV negative & $87(45.1 \%)^{\mathrm{a}}$ & $297(59.3 \%)^{\mathrm{a}}$ & $185(71.9 \%)^{\mathrm{a}}$ & $35(71.4 \%)^{\mathrm{a}}$ & $604(60.4 \%)^{t}$ \\
\hline HPV positive & $106(54.9 \%)^{\mathrm{a}}$ & $204(40.7 \%)^{\mathrm{a}}$ & $72(28 \%)^{\mathrm{a}}$ & $14(28.6 \%)^{\mathrm{a}}$ & $396(39.6 \%)^{\prime}$ \\
\hline Infection with single HPV type & $33(31.1 \%)^{\mathrm{b}}$ & $91(44.6 \%)^{\mathrm{b}}$ & $38(52.8 \%)^{\mathrm{b}}$ & $8(57.2 \%)^{\mathrm{b}}$ & $170(16.8 \%)^{\prime}$ \\
\hline Co-infection with multiple HPV types & $73(68.9 \%)^{\mathrm{b}}$ & $113(55.4 \%)^{\mathrm{b}}$ & $34(47.2 \%)^{\mathrm{b}}$ & $6(42.9 \%)^{\mathrm{b}}$ & $226(22.6 \%)^{\prime}$ \\
\hline All HR-HPV positive & $79(74.5 \%)^{\mathrm{b}}$ & $154(75.5 \%)^{\mathrm{b}}$ & $49(68.1 \%)^{\mathrm{b}}$ & $8(57.1 \%)^{\mathrm{b}}$ & $290 \quad(29 \%)^{\prime}$ \\
\hline HPV-16 & $32(30.2 \%)^{b}$ & $49(24 \%)^{\mathrm{b}}$ & $19(26.4 \%)^{\mathrm{b}}$ & $3(21.4 \%)^{\mathrm{b}}$ & $103(10.3 \%)^{\prime}$ \\
\hline HPV-18 & $15(14.2 \%)^{\mathrm{b}}$ & $15(7.4 \%)^{\mathrm{b}}$ & $4(5.5 \%)^{\mathrm{b}}$ & $0 \quad(0 \%)^{\mathrm{b}}$ & $34(3.4 \%)^{\prime}$ \\
\hline PHR-HPV positive & $31(29.3 \%)^{\mathrm{b}}$ & $30(14.7 \%)^{\mathrm{b}}$ & $9(12.5 \%)^{\mathrm{b}}$ & $4(28.6 \%)^{\mathrm{b}}$ & $74(7.4 \%)^{\prime}$ \\
\hline LR-HPV positive & $57(53.8 \%)^{\mathrm{b}}$ & $83(40.7 \%)^{\mathrm{b}}$ & $22(30.5 \%)^{\mathrm{b}}$ & $4(28.6 \%)^{\mathrm{b}}$ & $166(16.6 \%)^{\prime}$ \\
\hline Only LR-HPV positive & $10 \quad(9.4 \%)^{\mathrm{b}}$ & $21(10.3 \%)^{\mathrm{b}}$ & $10(13.8 \%)^{\mathrm{b}}$ & $3(21.4 \%)^{\mathrm{b}}$ & $44(4.4 \%)$ \\
\hline UR-HPV positive & $24(22.6 \%)^{\mathrm{b}}$ & $42(20.6 \%)^{\mathrm{b}}$ & $16(22.2 \%)^{b}$ & $4(28.6 \%)^{\mathrm{b}}$ & $86(8.6 \%)^{\prime}$ \\
\hline NILM & $141(73.05 \%)^{\mathrm{a}}$ & $408(81.4 \%)^{\mathrm{a}}$ & $213(82.8 \%)^{\mathrm{a}}$ & $39(79.6 \%)^{\mathrm{a}}$ & $801(80.1 \%)^{\prime}$ \\
\hline ASCUS & $26(13.5 \%)^{\mathrm{a}}$ & $45(8.9 \%)^{\mathrm{a}}$ & $33(12.8 \%)^{\mathrm{a}}$ & $4(8.1 \%)^{\mathrm{a}}$ & $108(10.8 \%)^{\prime}$ \\
\hline LSIL & $22(11.4 \%)^{\mathrm{a}}$ & $28(5.6 \%)^{\mathrm{a}}$ & $2(0.78 \%)^{\mathrm{a}}$ & $4(8.16 \%)^{\mathrm{a}}$ & $56(5.6 \%)^{\prime}$ \\
\hline HSIL & $4 \quad(4.3 \%)^{\mathrm{a}}$ & $20(3.9 \%)^{\mathrm{a}}$ & $9(3.5 \%)^{\mathrm{a}}$ & $2(4.08 \%)^{\mathrm{a}}$ & $35(3.5 \%)^{t}$ \\
\hline
\end{tabular}

*HR - High Risk, PHR - Probable High Risk, LR - Low Risk, UR - Undetermined Risk, NILM - Negative for Intraepithelial Lesions or Malignancy, ASCUS - Atypical Squamous Cells of Undetermined Significance, LSIL - Low Grade Squamous Intra-Epithelial Lesion, HSIL - High-Grade Intra-Epithelial Lesion; 'percentage of total,

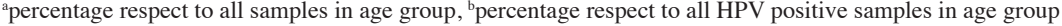

Table 2. Distribution of HPV Types in Infected Women by Cytological Result

\begin{tabular}{lcrrrr}
\hline & NILM & \multicolumn{1}{c}{ ASCUS } & LSIL & HSIL & Total (\%) \\
\hline HPV negative & $526(87.1 \%)^{\mathrm{d}}$ & $51(8.44 \%)^{\mathrm{d}}$ & $19(3.15 \%)^{\mathrm{d}}$ & $8(1.32 \%)^{\mathrm{d}}$ & $604(60.4 \%)^{\mathrm{t}}$ \\
HPV positive & $275(69.4 \%)^{\mathrm{d}}$ & $57(14.4 \%)^{\mathrm{d}}$ & $37(9.34 \%)^{\mathrm{d}}$ & $27(6.81 \%)^{\mathrm{d}}$ & $396(39.6 \%)^{\mathrm{t}}$ \\
Single HPV infection & $130(76.5 \%)^{\mathrm{d}}$ & $23(13.5 \%)^{\mathrm{d}}$ & $7(4.11 \%)^{\mathrm{d}}$ & $10(5.88 \%)^{\mathrm{d}}$ & $170(42.9 \%)^{\mathrm{c}}$ \\
Coinfection HPV & $145(64.2 \%)^{\mathrm{d}}$ & $34(15.04 \%)^{\mathrm{d}}$ & $30(13.3 \%)^{\mathrm{d}}$ & $17(7.5 \%)^{\mathrm{d}}$ & $226(57.1 \%)^{\mathrm{c}}$ \\
All HR-HPV & $194(66.9 \%)^{\mathrm{d}}$ & $45(15.5 \%)^{\mathrm{d}}$ & $30(10.3 \%)^{\mathrm{d}}$ & $21(7.24 \%)^{\mathrm{d}}$ & $290(73.2 \%)^{\mathrm{c}}$ \\
HPV-16 & $60(58.3 \%)^{\mathrm{d}}$ & $19(18.4 \%)^{\mathrm{d}}$ & $13(12.6 \%)^{\mathrm{d}}$ & $11(10.7 \%)^{\mathrm{d}}$ & $103(26 \%)^{\mathrm{c}}$ \\
HPV-18 & $21(61.8 \%)^{\mathrm{d}}$ & $6(17.6 \%)^{\mathrm{d}}$ & $5(14.7 \%)^{\mathrm{d}}$ & $2(5.8 \%)^{\mathrm{d}}$ & $34(8.58 \%)^{\mathrm{c}}$ \\
PHR-HPV & $52(70.3 \%)^{\mathrm{d}}$ & $14(18.9 \%)^{\mathrm{d}}$ & $3(4.05 \%)^{\mathrm{d}}$ & $5(6.75 \%)^{\mathrm{d}}$ & $74(18.7 \%)^{\mathrm{c}}$ \\
LR-HPV & $122(73.5 \%)^{\mathrm{d}}$ & $19(11.5 \%)^{\mathrm{d}}$ & $15(9.03 \%)^{\mathrm{d}}$ & $10(6.02 \%)^{\mathrm{d}}$ & $166(41.9 \%)^{\mathrm{c}}$ \\
Only LR-HPV & $38(86.4 \%)^{\mathrm{d}}$ & $3(6.8 \%)^{\mathrm{d}}$ & $2(4.54 \%)^{\mathrm{d}}$ & $1(2.27 \%)^{\mathrm{d}}$ & $44(11.1 \%)$ \\
UR-HPV & $59(68.6 \%)^{\mathrm{d}}$ & $11(12.8 \%)^{\mathrm{d}}$ & $7(8.14 \%)^{\mathrm{d}}$ & $9(10.5 \%)^{\mathrm{d}}$ & $86(21.7 \%)^{\mathrm{c}}$ \\
& $801(80.1 \%)^{\mathrm{t}}$ & $108(10.8 \%)^{\mathrm{t}}$ & $56(5.6 \%)^{\mathrm{t}}$ & $35(3.5 \%)^{\mathrm{t}}$ & $1000(100 \%)^{\mathrm{t}}$ \\
\hline
\end{tabular}

*HR - High Risk, PHR - Probable High Risk, LR - Low Risk, UR - Undetermined Risk, NILM - Negative for Intraepithelial Lesions or Malignancy, ASCUS - Atypical Squamous Cells of Undetermined Significance, LSIL - Low Grade Squamous Intra-Epithelial Lesion, HSIL - High-Grade Intra-Epithelial Lesion; 'percentage of total, cpercentage respect to all positive samples, ${ }^{\mathrm{d}}$ percentage respect to all positive samples in infection group.

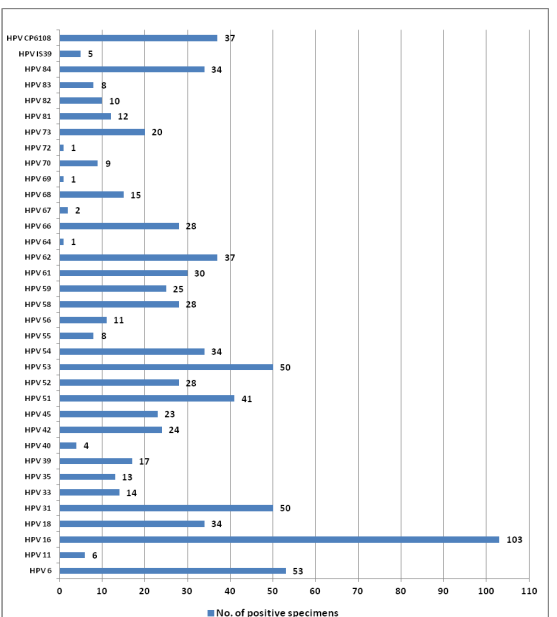

Figure 1. Distribution of HPV Genotypes in Infected Women

HPV types in order of frequency were HPV-16 (26.01\%), HPV-6 (13.38\%), HPV-31 and HPV-53 (12.26\%), HPV-51 (10.35\%), HPV-62 and HPV-CP6108 (9.34\%), HPV-18, HPV-54 and HPV-84 (8.58\%) (Figure 1).

HPV infection prevalence was the highest in young women and decreased with age $(\mathrm{p}<0.0001)$. Women aged under 25 years had the highest prevalence of HPV (54.93\%), followed by women aged $26-35$ years with a prevalence of $40.72 \%$, women aged over 45 years with a prevalence of HPV infection of $28.58 \%$ and the lowest prevalence of $28.02 \%$ in women aged between 36 and 45 years (Table 1). Regardless of the age, the HR-HPV types were the most frequent $(73.23 \%$ of overall HPV prevalence) with the highest prevalence $(75.5 \%)$ in females between 26 and 35 years subsequently showing an age-dependent decrease $(\mathrm{p}<0.001)$. In contrast, the proportion of only LR-HPV infection showed a significant age-specific increase $(9.4 \%, 10.3 \%, 13.8 \%$ and $21.4 \%$, respectively; $\mathrm{p}<0.005)$. There was a peak in PHR-HPV infection rate among women aged less than 25 years $(29.25 \%)$ with a second peak among the oldest age group $(28.57 \%)$. The prevalence of UR-HPV infection remained approximately constant in all age groups $(\mathrm{p}<0.0005)$.

Infection with a single HPV type was noted in $42.93 \%$ of cases (170 out of 396 positive samples) and coinfection with multiple HPV genotypes (up to 9 different HPV types in a single sample) in $57.07 \%$ of cases (226 out of 396 positive samples). It was noted a peak in HPV coinfection 
rate among women under 25 years $(68.87 \%)(\mathrm{p}<0.005)$. One HR-HPV types was noted in 16 cases, multiple combinations of HR-HPV types were found in 20 cases, thus: 2 HR-HPV types in 15 cases, 3 HR-HPV types in 3 cases and in one case each 4 and 5 HR-HPV types. The most common HR-HPV type seen in combinations in was HPV-16.

The overall prevalence of abnormal cervical cytology (ASCUS, LSIL, HSIL) was 19.9\%. The highest prevalence of abnormal cervical cytology was in the youngest group (26.95\%) and the lowest was in the 36 to 45 -year-old group (17.12\%). Table 2 shows the distribution of HPV types in infected women by cytological result. The prevalence of HR-HPV types by cervical cytological changes was calculated regardless of multiple infections (one or more HR-HPV types). Among $396 \mathrm{HPV}$ positive patients, 121 cases $(30.55 \%)$ had abnormal cytology. 96 of 199 (48.24\%) women with abnormal cytology were infected with HR-HPV types, whereas less than $3 \%$ of them were infected only with LR-HPV types. The prevalence of ASCUS, LSIL, and HSIL was 15.5\%, 10.34\%, and 7.24\% in patients infected with HR-HPV types and 6.8\%, $4.54 \%$, and $2.27 \%$ in patients infected only with LR-HPV types, respectively. The prevalence of HR-HPV types among HPV positive samples was $78.9 \%, 81.08 \%$, and $77.7 \%$ in patients with ASCUS, LSIL and HSIL, respectively. In the HPV positive group, the odds ratio for detection of abnormal cytology was 3.62 (95\% CI, 1.49 - 8.8) for HR-HPV positive patients in comparison to patients infected with only LR-HPV types $(\mathrm{p}<0.005)$. In women infected by HPV-16 or HPV-18 was observed the highest prevalence of cervical cytological abnormalities (Table 2). HPV coinfection rate in women with abnormal cytology had a peak for LSIL (81.08\%) and declined from ASCUS to HSIL (66.67\% to $62.96 \%$ ).

\section{Discussion}

Oncogenic role of HPV is clearly shown in triggering of CC (Walboomers et al., 1999). Among asymptomatic women, the prevalence of HPV infection ranges between 2 and 44\% (Trottier and Franco, 2006). To date 15 oncogenes HPV types $(16,18,31,33,35,39,45,51,52$, $56,58,59,68,73$, and 82) considered of high risk for CC have been identified (Meijer et al., 2006). HPV16 and 18 are responsible for over $70 \%$ of all of them (Smith et al., 2007; Pandey et al., 2012).

In our study population the overall prevalence of infections with any HPV type $(39.6 \%)$ and prevalence of HR-HPV (29\%) were higher in comparison with other studies (Masia et al., 2009; Lee et al., 2012). The six most prevalent HR-HPV types were HPV-16, 31, 51, 18, 52 and 58 in descending orders. These six most common HR-HPV genotypes accounted $34.71 \%$ of all HPV genotypes (284 out of 818 specific-type infections) and $68.1 \%$ of all HRHPV genotypes (284 out of 417 specific-type infections). Our findings on HPV-16 infection (26.01\%) were also higher to that reported worldwide (Clifford et al., 2005; Masia et al., 2009). Prevalence rates worldwide for HPV16 tend to be higher than those observed for other HPV types (Trottier and Franco, 2006; Hamzi Abdul Raub et al.,
2014; Siriaunkgul et al. 2014). The second most frequent HR-HPV type was HPV-31 (12.26\%), unlike HPV-18 in Europe, Central and South America, HPV-52 and 58 in Asia, and HPV-52 and 53 in North America (Clifford et al., 2005; De Sanjose et al., 2010). These differences in HPV type distribution in different areas may be related to different sexual habits and migrations of population (Bosch et al., 1995; Barzon et al., 2008; Natphopsuk et al., 2013). According to the most studies prevalence rates, with varying estimates, are generally higher for HR-HPV types than for LR-HPV types (Smith et al., 2008; Othman and Othman, 2014). In our study, $73.23 \%$ of infected women presented HR-HPV types similar to the most populationbased studies where HR-HPV types were detected in greater than $70 \%$ of all HPV positive women. Coinfection with multiple HPV types was observed in $57.07 \%$ of cases in most of them being present a HR-HPV. The prevalence of multiple HPV infections in HPV positive patients has shown a geographical variation ranging from about $9 \%$ to $50 \%$ in the European countries (Bosch et al., 1995; Forslund et al., 2002). The risk of acquiring new HPV types appears to be independent of prior infection with other types (Trottier; Franco, 2006).

Our findings document that HPV infection is the most frequent in women aged under 25 years, then a new slight increase in prevalence of HPV infection was observed in women aged over 45 years. The majority of epidemiological studies provide evidence that genital HPV infection is very common in young sexually active women with prevalences up to 76-80\% (Weinstock et al., 2004; Brown et al., 2005). This is the age which is most sexually active. Then we noted a gradual decrease in incidence of HPV infection up to the age of 45 years. This pattern is observed in the most studies all over the world, although second peaks are sometimes observed in older women (Munoz et al., 2004; Franceschi et al., 2006; Trottier and Franco, 2006; Lee et al., 2012). This decrease in HPV infection prevalence with age may be explained by the fact in most cases HPV infection is self-limited and the virus is cleared by the host innate immune response (Ho et al., 1998; Stanley, 2006; Datta et al., 2012). In some cases the immune response fails to clear the infection and in subjects persistently infected by HR-HPV types there is a risk of progression to high-grade cervical intraepithelial neoplasia and invasive cancer (Ho et al., 1998; Schlecht et al., 2001; Snijders et al., 2006). HPV infections in older women do not seem to represent new infections but are long lasting infections which require more attention and a closer follow up because of the increased risk of CC related to the length of infection (Masia et al., 2009).

An increasing trend in overall prevalence of HPV infection as well as prevalence of HR-HPV was observed in parallel with increasing the degree of cervical cytological abnormalities. About $60.8 \%$ of the women with a positive cytological examination (including ASCUS, LSIL, and HSIL) were infected by any HPV and over $28.64 \%$ of them were infected by an HR-HPV type. The high presence of HR-HPV genotypes in precancerous lesions emphasizes the need of a more frequent follow up in women who are HR-HPV positive. However, HPV tests are not sufficient in surveillance for CC since the test was negative in $39.2 \%$ 
of patients with abnormal cytology and therefore HPV follow up would have missed almost $40 \%$ of the negative HPV women with an abnormal Pap smear test result requiring a cytological surveillance. It was also shown a significantly higher proportion of abnormal cytology among women infected by HPV-16 or HPV-18, HPV genotypes which are included in existing vaccine. From our findings results that the use of vaccines containing HPV-16 and 18 genotypes would only prevent $20.7 \%$ of infections in women younger than 25 years. However, the prevalence of cervical cytological abnormalities among women infected with HR-HPV types, other than HPV-16 or HPV-18, remains increased. Many studies reported that the risk for developing the cytological abnormalities or lesions that typically precede $\mathrm{CC}$ is much higher in women infected with multiple genotypes of the HPV (Fife et al., 2001; Bello et al., 2009) . HPV coinfection rate in women with abnormal cytology had a peak for LSIL and declined from ASCUS to HSIL. In most similar studies the highest coinfection rate was reported in women with cervical cytology of ASCUS (Castellsague et al., 2001) or LSIL (Herrero et al., 2000).

As it is evident that early detection can greatly reduce death by $\mathrm{CC}$ as it can be treated if infection is found in early stages. So a coherent policy about the spread of awareness about HPV infection is absolutely necessary which can stop the spread the $\mathrm{CC}$ by detecting the infection at early stage help physicians for a better patient management. Our data suggest the need to integrate both HPV-DNA genotyping and Pap test, the complementary use of the two tests providing an assessment both of the virological and cytological risk.

Our study showed that the prevalence of HR-HPV infection among Romanian women is higher compared to other studies in other geographic areas. Thus, we consider that in areas where there is an increased prevalence of high-risk HPV infections HPV genotyping should be performed in all women aged between 18 and 45 years, and Pap test should be carried out every 6 months in women with high-risk HPV infection even those with previous normal cervical cytology. The higher prevalence of cervical cytological abnormalities in Romanian women infected with high risk HPV types which are not included in the existing vaccines should be considered in future immunization programs. However, our findings have some limitations given that the patients included in the study are women who referred to our outpatient clinic for various genital symptoms and the prevalence results presented in this study could overestimate the cumulative HPV incidence thereby other larger epidemiological studies are required.

\section{References}

Aral SO, Holmes KK (1999). Social and behavioral determinants of epidemiology of STDs: industrialized and developing countries. In: Holmes KK, Mardh P-A, Sparling PF, et al., editors. Sexually Transmitted Diseases. New York: McGrawHill; p. 39-76.

Barzon L, Giorgi C, Buonaguro FM, Palu G (2008). Guidelines of the Italian Society for Virology on HPV testing and vaccination for cervical cancer prevention. Infect Agents Cancer, 3, 14.

Bello BD, Spinillo A, Alberizzi P, et al (2009). Cervical infections by multiple human papillomavirus (HPV) genotypes: prevalence and impact on the risk of precancerous epithelial lesions. J Med Virol, 81, 703-12.

Bosch FX, Manos MM, Munoz N, et al (1995). International biological study on cervical cancer (ibscc) study group. prevalence of human papillomavirus in cervical cancer: a worldwide perspective. J Natl Cancer Inst, 87, 796-802.

Bouvard V, Baan R, Straif K, et al (2009). A review of human carcinogens - Part B: biological agents. Lancet Oncol, 10, 321-2.

Brown DR, Shew ML, Qadadri B, et al (2005). A longitudinal study of genital human papillomavirus infection in a cohort of closely followed adolescent women. J Infect Dis, 191, 182-92.

Castellsague X, Menendez C, Loscertales MP, et al (2001). Human papillomavirus genotypes in rural Mozambique. Lancet, 358, 1429-30.

Clifford GM, Gallus S, Herrero R, et al (2005). Worldwide distribution of human papillomavirus types in cytologically normal women in the International Agency for Research on Cancer HPV prevalence surveys: a pooled analysis. Lancet, 366, 991-8.

Clifford GM, Smith JS, Plummer M, Munoz N, Franceschi S (2003). Human papillomavirus types in invasive cervical cancer worldwide: A metaanalysis. Br J Cancer, 88, 63-73.

Datta P, Bhatla N, Pandey RM, et al (2012). Type-specific incidence and persistence of HPV infection among young women: a prospective study in North India. Asian Pac J Cancer Prev, 13, 1019-24.

Davey DD, Neal MH, Wilbur DC, et al (2004). Bethesda 2001 implementation and reporting rates: 2003 practices of participants in the college of american pathologists interlaboratory comparison program in cervicovaginal cytology. Arch Pathol Lab Med, 128, 1224-9.

De Sanjose S, Quint WC, Alemany L, et al (2010). Human papillomavirus genotype attribution in invasive cervical cancer: a retrospecttive cross-sectional worldwide study. Lancet Oncol, 11, 1048-56.

Fife KH, Cramer HM, Schroeder JM, Brown DR (2001). Detection of multiple human papillomavirus types in the lower genital tract correlates with cervical dysplasia. J Med Virol, 64, 550-9.

Forslund O, Antonsson A, Edlund K, et al (2002). Populationbased type-specific prevalence of high-risk human papillomavirus infection in middle-aged Swedish women. J Med Virol, 66, 535-41.

Franceschi S, Herrero R, Clifford GM, et al (2006). Variations in the age-specific curves of human papillomavirus prevalence in women worldwide. Int J Cancer, 119, 2677-84.

GLOBOCAN 2008 (IARC), Section of Cancer Information (http://globocan.iarc.fr/Default.aspx). Accesed 12 March 2014.

Halfon P, Sandri MT, Raimondo A, et al (2013). Comparison of the performance of carcinogenic HPV typing of the Roche Linear Array and Qiagen LiquiChip ${ }^{\circledR}$ HPV assays. BMC Infect Dis, 13, 499.

Hamzi Abdul Raub S, Isa NM, Zailani HA, et al (2014). Distribution of HPV genotypes in cervical cancer in multiethnic Malaysia. Asian Pac J Cancer Prev, 15, 651-6.

Herrero R, Hildesheim A, Bratti C, et al (2000). Populationbased study of human papillomavirus infection and cervical neoplasia in rural Costa Rica. J Natl Cancer Inst, 92, 464-74.

Ho GY, Bierman R, Beardesley L, et al (1998). Natural history of cervicovaginal papillomavirus infection in young women. 
N Engl J Med, 338, 423-8.

IARC monographs on the evaluation of carcinogenic risks to humans: A review of human carcinogens: part B. Biological agents. 100th edition. Lyon, France: International Agency for Research on Cancer; 2011: 261-319. http://monographs.iarc. fr/ENG/Monographs/vol100B/mono100B-11.pdf .

Lee EH, Um TH. Chi HS, et al (2012). Prevalence and distribution of human papillomavirus infection in Korean women as determined by restriction fragment mass polymorphism assay. J Korean Med Sci, 27, 1091-7.

Mandelblatt JS, Lawrence WF, Womack SM, et al. (2002). Benefits and costs of using HPV testing to screen for cervical cancer. JAMA, 287, 2372-81.

Masia G, Mazzoleni AP, Contu G, et al (2009). Epidemiology and genotype distribution of human papillomavirus (HPV) in women of Sardinia (Italy). Vaccine, 27, 11-16.

Meijer CJ, Snijders PJ, Castle PE (2006). Clinical utility of HPV genotyping. Gynecol Oncol, 103, 12-17.

Munoz N, Mendez F, Posso H, et al (2004). Incidence, duration, and determinants of cervical human papillomavirus infection in a cohort of Colombian women with normal cytological results. J Infect Dis, 190, 2077-87.

Natphopsuk S, Settheetham-Ishida W, Pientong C, et al (2013). Human papillomavirus genotypes and cervical cancer in northeast Thailand. Asian Pac J Cancer Prev, 14, 6961-4.

Nessa A, Nahar KN, Begum SA, et al. (2013). Comparison between visual inspection of cervix and cytology based screening procedures in Bangladesh. Asian Pac J Cancer Prev, 14, 7607-11.

Othman N, Othman NH (2014). Detection of human papillomavirus DNA in routine cervical scraping samples: use for a national cervical cancer screening program in a developing nation. Asian Pac J Cancer Prev, 15, 2245-9.

Pandey S, Mishra M, Chandrawati (2012). Human papillomavirus screening in north Indian women. Asian Pac J Cancer Prev, 13, 2643-6.

Schlecht NF, Kulaga S, Robitaille J, et al (2001). Persistent human papillomavirus infection as a predictor of cervical intraepithelial neoplasia. JAMA, 286, 3106-14.

Sengul D, Altinay S, Oksuz H, Demirturk H, Korkmazer E (2014). Population-based cervical screening outcomes in Turkey over a period of approximately nine and a half years with emphasis on results for women aged 30-34. Asian Pac J Cancer Prev, 15, 2069-74.

Siriaunkgul S, Settakorn J, Sukpan K, et al (2014). HPV detection and genotyping in vulvar squamous cell carcinoma in northern Thailand. Asian Pac J Cancer Prev, 15, 3773-8.

Smith JS, Lindsay L, Hoots B, et al (2007). Human papillomavirus type distribution in invasive cervical cancer and high-grade cervical lesions: a meta-analysis update. Int J Cancer, 121, 621-32.

Smith JS, Melendy A, Rana RK, Pimenta JM (2008). Agespecific prevalence of infection with human papillomavirus in females: a global review. J Adolesc Health, 43, 5-25.

Snijders PJ, Steenberg RD, Heideman DA, Meijer CJ (2006). HPV-mediated cervical carcinogenesis: concepts and clinical implications. J Pathol 208, 152-64.

Stanley M (2006). Immune responses to human papillomavirus. Vaccine, 24, 16-22.

Trottier H, Franco EL (2006). The epidemiology of genital human papillomavirus infection. Vaccine, 24, 4-15.

Veena S, Premraj S, Narotam S, Pracheta, Ritu P (2012). Study of epidemiology of HPV infection in the uterine cervix of women in Delhi /NCR regions, India. Int J Drug Development and Research, 4, 311-5.

Wang SM, Zhang SK, Pan XF, et al (2014). Human papillomavirus vaccine awareness, acceptability, and decision-making factors among chinese college students. Asian Pac J Cancer Prev, 15, 3239-45.

Wang YY, Li L, Wei S, et al (2013). Human papillomavirus (HPV) infection in women participating in cervical cancer screening from 2006 to 2010 in Shenzhen City, South China. Asian Pac J Cancer Prev, 14, 7483-7.

Weinstock H, Berman S, Caces Jr W (2004). Sexually transmitted diseases among American youth: incidence and prevalence estimates, 2000. Perspect Sex Reprod Health, 36, 6-10. 